\title{
Positive magnetic susceptibility in polygonal nanotube tori
}

\author{
Ryo Tamura \\ Faculty of Engineering, Shizuoka University, Johoku 3-5-1, Hamamatsu 432-8561, Japan \\ Mitsuhiro Ikuta and Toru Hirahara \\ Department of Physics, Graduate School of Science, University of Tokyo, Hongo 7-3-1, Bunkyo-ku, Tokyo 113-0033, Japan \\ Masaru Tsukada \\ Department of Nano-science and Nano-engineering, Graduate School of Science and Engineering, Waseda University, \\ 513 Waseda Tsurumaki-cho, NTRC bldg. 120-5, Shinjuku-ku, Tokyo, 162-0041, Japan \\ (Received 17 December 2003; revised manuscript received 1 July 2004; published 14 January 2005)
}

\begin{abstract}
A type of large paramagnetic persistent current is revealed in polygonal carbon nanotube (CNT) tori through calculation of the persistent current-induced magnetic moment using a tight binding model. Polygonal CNT tori, constructed by apices formed at heptagonal and pentagonal defects on the inner and outer fringes, exhibit paramagnetism related to the semimetallic band structures of the periodic CNT junctions, despite the inherent diamagnetism of graphite. This type of persistent current differs from that originating from metallic band structures, and is caused by both magnetic flux in the inner hole (Aharonov-Bohm flux) and the magnetic flux penetrating the graphite plane directly (direct flux). This magnetic moment is close to that calculated based on the Aharonov-Bohm alone for a cross section that includes the region of direct flux.
\end{abstract}

DOI: 10.1103/PhysRevB.71.045418 PACS number(s): 73.63.Fg, 73.23.Ra, 85.35.Kt, 73.22. $-\mathrm{f}$

\section{INTRODUCTION}

Despite the semimetallic nature of graphite, carbon nanotubes (CNTs) become metallic and semiconducting depending on the radius and helicity of the honeycomb lattice. ${ }^{1}$ This promising feature is caused by the quantum size effect, whereby new electronic states arise as the system becomes smaller than the coherent length. Such quantum size effects have also been identified in the persistent current of the mesoscopic ring; an ideal clean, one-dimensional, mesoscopic metal ring is diamagnetic when the number of electrons is $4 n+2$ for integer $n$, and paramagnetic otherwise. ${ }^{2}$ This means that the ring can have the opposite sign of magnetic susceptibility compared to the bulk system. Furthermore, this positive magnetic susceptibility is inversely proportional to temperature, becoming divergent at absolute zero. ${ }^{2-4} \mathrm{Al}-$ though this appears superficially similar to the Curie-Weiss law, the origin of this temperature dependence is not spin but persistent current. In the absence of generally accepted terminology for this type of paramagnetism, it is referred to as giant orbital paramagnetism (GOP) in this paper.

Our group has become interested in the recently discovered CNT ring for a number of reasons. ${ }^{5}$ First, similar to graphite, CNTs exhibits negative values of magnetic susceptibility with respect to the magnetic field perpendicular tothe honeycomb plane, ${ }^{6}$ yet the persistent current of the CNT ring can also produce GOP. ${ }^{4}$ Furthermore, CNT rings are more suitable for miniaturization to a nanometer-scale radius $R$ than the conventional mesoscopic rings of metals or GaAs/AlGaAs. ${ }^{7}$ Such miniaturization is desirable from two perspectives. (1) The circulating persistent current $I$ is given in terms of the Fermi velocity $v_{F}$ by $e v_{F} /(2 \pi R)$, and is also related to the magnetic moment per ring $M$ by $M=I \pi R^{2}$. Therefore, in closed-packed rings, the magnetic moment per unit area is proportional to $I$ and can be increased by shrink- ing $R$. Additionally, (2) the persistent current occurs on the condition that the coherent length $l_{\phi}$ is larger than $2 \pi R$. Since $l_{\phi}$ decreases with increasing temperature, the persistent current can be observed at higher temperature as $R$ decreases.

In other theoretical studies on the persistent current of the CNT ring, "disclination" and "direct flux" have not been considered together. ${ }^{4,8-11}$ In the present study, however, both are considered, as reducing the radius of the CNT ring increases the importance of these two factors. The effect of disclination is illustrated schematically in Fig. 1. The polygonal CNT torus in the figure is formed by pentagonal and heptagonal defects that produce the corners of the polygonal shape on the outer and inner fringes, respectively. These defects are generally called disclinations and have been shown

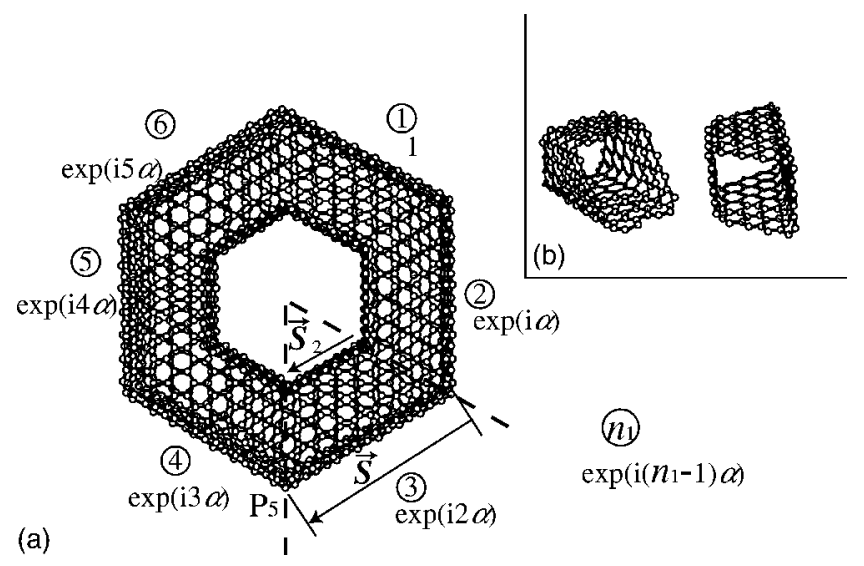

FIG. 1. (a) Schematic of three-dimensional structure of a polygonal CNT torus. Uniform magnetic field is applied perpendicular to the page. Unit cell labels are denoted by $n_{1}$. (b) Unit cell of the CNT torus. The torus is composed of six unit cells. 
to have a significant effect on CNT junctions, ${ }^{12} \mathrm{CNT}$ caps, ${ }^{13}$ and helical CNTs. ${ }^{14,15}$ Here, a polygonal CNT torus is defined as a CNT torus with this nature of disclinations, although more rounded shapes can also be achieved by a series of more closely spaced disclinations. ${ }^{16}$

Only a few attempts have so far been made to investigate the persistent current of the polygonal CNT torus, ${ }^{17}$ whereas the circular CNT torus has been discussed by many authors. ${ }^{4,8-11}$ A circular CNT torus is defined here as a CNT torus formed by elastic deformation of the straight CNT with no disclination. The elastic energy of the circular CNT torus has been investigated theoretically. ${ }^{18}$ In the circular CNT torus, the honeycomb lattice shrinks along the tube axis by a factor of $R_{1} /\left(R_{1}+D\right)$ on the inner fringe compared to the outer fringe $\left(R_{1}\right.$ and $D$ denote the radius of the inner hole and the diameter of the CNT, respectively). Although the resultant axial strain is relaxed to a certain degree by the elastic deformation known as "buckling," 19 the relaxation is not sufficient to maintain stability if $R_{1}$ approaches $D$. As shown in Fig. 1, however, the strain in the polygonal CNT torus can be relaxed by reducing the number of hexagons on the inner fringe.

It is widely known that impurities suppress the GOP effect, ${ }^{3,8}$ but the effect of disclinations on GOP remains open for discussion. The effect of "direct flux" on GOP also requires further attention. Previously, only the magnetic flux in the inner hole, that is, flux that induces the Aharonov-Bohm effect ( $\mathrm{AB}$ flux), has been considered. In this study, however, we also consider the magnetic flux which contacts the electrons in the graphite plane directly (direct flux). The ratio of $\mathrm{AB}$ flux to direct flux is given by $R_{1}^{2}$ to $\left(R_{1}+D\right)^{2}-R_{1}^{2}$, suggesting that the direct flux cannot be neglected when $R_{1}$ is comparable to $D$. As graphite is diamagnetic under direct flux, it is necessary to determine whether direct flux reduces GOP. These areas of uncertainty are clarified in this study by investigating the influence of disclination and direct flux on the magnetic moment of the polygonal CNT torus.

\section{TIGHT BINDING MODEL}

A polygonal CNT torus composed entirely of semiconducting CNTs has a finite HOMO-LUMO gap (highest occupied-lowest unoccupied molecular orbital). It is shown later that GOP does not appear under this condition. Thus, the discussion can be limited hereto metallic CNTs. The first example considered is the polygonal CNT torus with $D_{6 h}$ symmetry formed from armchair CNTs, which are metallic irrespective of radius. The tight binding (TB) model with exclusively $\pi$ orbitals is employed, and the positions of atoms are given by the assumed polygonal shape of the torus.

Figure 2 shows a part of the projection map for a polygonal CNT torus. The torus is composed of six unit cells, one of which is represented by the rectangle $A B B^{\prime} A^{\prime}$. The prime indicates that points $X^{\prime}$ and $X$ become identical when the three-dimensional shape is formed. Vector $\vec{L}$ denotes the circumference of the original armchair CNT. The length and direction of the CNT axis per unit cell along the outer and inner fringes is represented by $\vec{S}$ and $\vec{S}_{2}$, respectively. The

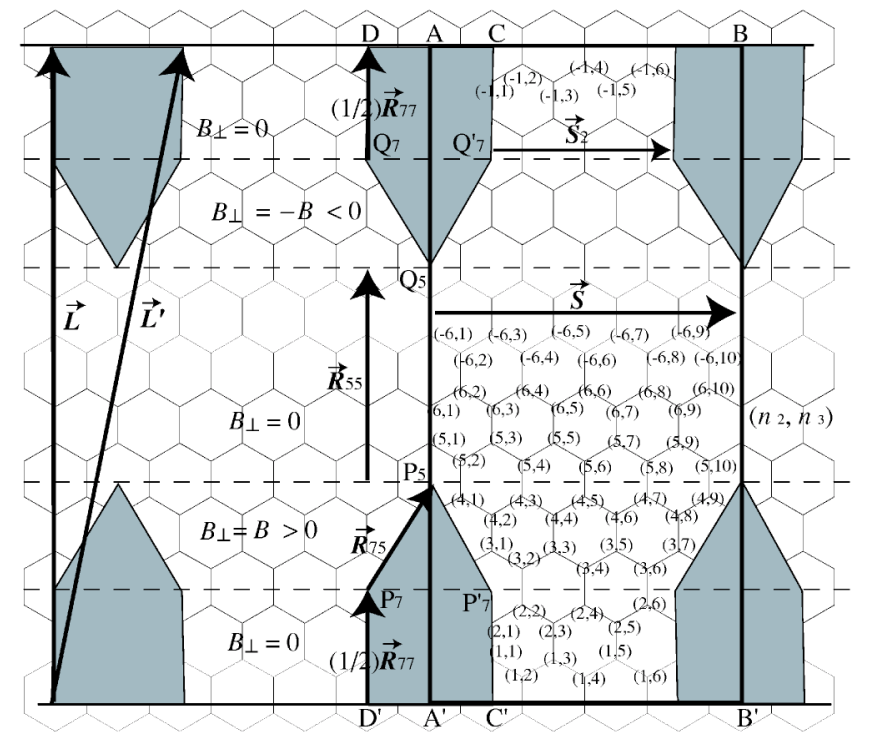

FIG. 2. Projection map for the CNT torus. The rectangle $A$ to $B^{\prime}$ is the unit cell. Magnetic field is applied perpendicular to the page $\left(B_{\perp}\right)$. Atoms $\left(n_{2}, n_{3}\right)$ in the unit cell are labeled.

$i$-membered ring disclination is formed at $P_{i}$ and $Q_{i}$ $(i=5,7)$ by removal of the shaded area and attaching the lines $P_{5} P_{7}, P_{7} D^{\prime}, D Q_{7}$, and $Q_{7} Q_{5}$ to the lines $P_{5} P^{\prime}{ }_{7}, P^{\prime}{ }_{7} C^{\prime}$, $C Q^{\prime}{ }_{7}$, and $Q^{\prime}{ }_{7} Q_{5}$, respectively. The disclinations approximately maintain the $s p^{2}$ bonds of carbon atoms (three nearest neighbors for each atom). Despite the unchanged local structure, the phase of the wave function is shifted when an electron circulates around the disclination due to an excess or deficiency of the $\pi / 3$ angle on the projection map. This topological effect cannot be represented by the effective potential energy or by modification of the bond strength. ${ }^{20}$

As a result of the $D_{6 h}$ symmetry, $\vec{R}_{55}$ and $\vec{R}_{77}$ are parallel to $\vec{L}$, and $\vec{S}_{2}$ and $\vec{S}$ are perpendicular to $\vec{L}$. The definition of $\vec{R}_{i, j}$ is illustrated in Fig. 2. The four integer parameters, $n_{L}$ $=|\vec{L}| /(\sqrt{3} a), n_{75}=\left|\vec{R}_{75}\right| / a, n_{S}=|\vec{S}| / a$, and $n_{77}=\left|\vec{R}_{77}\right| /(\sqrt{3} a)$ define the CNT torus with a lattice constant of $a \simeq 0.25 \mathrm{~nm}$ for graphite. For example, the parameters in Fig. 2 are $n_{L}=6$, $n_{S}=5, n_{75}=2$, and $n_{77}=2$. To maintain almost constant bond lengths, the cross-section across $P_{5} P_{7} Q_{7} Q_{5} P_{5}$ must be rectangular, and such the four integers must satisfy the condition $n_{77}=\left(n_{L}-n_{75}\right) / 2$. A uniform magnetic field $\vec{B}$ parallel to the sixfold rotational axis of the torus is employed. In Fig. 2, $B_{\perp}$ denotes the component of $\vec{B}$ perpendicular to the projection map.

The unit cells are numbered along the CNT axis as shown in Fig. $1\left(n_{1}=1,2, \ldots, 6\right)$. Figure 2 shows the labels of atoms in each unit cell, $\left(n_{2}, n_{3}\right)$, where $n_{2}$ specifies the zigzag rows along the tube axis. On the inner hole side, $n_{2}=1$ and increases (or $n_{2}=-1$ and decreases) monotonically as the zigzag row ascends (descends) the INNER hole wall and approaches the outer fringe. In the $n_{2}$ zigzag row, atoms are numbered along the CNT axis as $n_{3}=1,2, \cdots, q\left(n_{2}\right)$. For example, $q(1)=q(2)=6, q(3)=7$ in Fig. 2. Between neighboring unit cells, atoms $\left(n_{1}, n_{2}, 1\right)$ and $\left(n_{1}, n_{2}, q\left(n_{2}\right)\right)$ connect to atoms $\left(n_{1}-1, n_{2}, q\left(n_{2}\right)\right)$ and $\left(n_{1}+1, n_{2}, 1\right)$, respectively. Us- 
ing this labeling scheme, the matrix elements of the Hamiltonian between atoms $n$ and $m$ are represented by

$$
H(B)_{n, m}=-t_{n, m} \exp (i \beta(n, m) B) .
$$

Each atom $n$ has a constant hopping integral $-t_{n, m}$ $=-t(\sim-3 \mathrm{eV})$ with the three nearest neighbors $m$, and $t_{n, m}$ $=0$ with all other atoms $m$. The effect of the magnetic fields is included in the form of Peierls phases $\beta(n, m) B,{ }^{21}$ where

$$
\begin{gathered}
\beta(n, m)=\gamma_{D}(n, m) C_{1}+\gamma_{A B}(n, m) C_{2}, \\
C_{1}=\sqrt{3} \pi a^{2} /\left(2 \phi_{0}\right),
\end{gathered}
$$

$$
C_{2}=\sqrt{3} \pi\left|\vec{S}_{2}\right|^{2} /\left(2 \phi_{0}\right)
$$

$$
\begin{gathered}
\gamma_{A B}(n, m)=\delta_{n_{2}, m_{2}}\left(\delta_{n_{1}, m_{1}+1} \delta_{n_{3}, 1} \delta_{m_{3}, q\left(n_{2}\right)}-\delta_{n_{1}, m_{1}-1} \delta_{m_{3}, 1} \delta_{n_{3}, q\left(n_{2}\right)}\right), \\
\gamma_{D}(n, m)= \\
\delta_{n_{2}, m_{2}}\left\{f ( n _ { 2 } ) \left(\delta_{q\left(n_{2}\right) n_{1}+n_{3}, q\left(n_{2}\right) m_{1}+m_{3}+1}\right.\right. \\
\left.\left.-\delta_{q\left(n_{2}\right) n_{1}+n_{3}, q\left(n_{2}\right) m_{1}+m_{3}-1}\right)+g\left(n_{2}\right) \gamma_{A B}(n, m)\right\} .
\end{gathered}
$$

Here, $\phi_{0}=h / e$ is the magnetic flux quantum, and $\delta$ is the Kronecker delta. The parameters $f$ and $g$ are constants or linear functions of $n_{2}$, as given by

$$
\begin{gathered}
f\left(n_{2}\right)=\left\{\begin{array}{l}
n_{75}, \quad \cdots\left|n_{2}\right| \geqslant 1+n_{75}+n_{77}, \\
\left|n_{2}\right|-n_{77}-0.5, \quad \cdots n_{77}+1 \leqslant\left|n_{2}\right| \leqslant n_{75}+n_{77},
\end{array}\right. \\
g\left(n_{2}\right)=\left\{\begin{array}{l}
-2 \sum_{j=1}^{n_{77}+n_{75}} f(j), \quad \cdots\left|n_{2}\right| \geqslant 1+n_{75}+n_{77}, \\
(1 / 4)-f\left(n_{2}\right)-2 \sum_{j=1}^{\left|n_{2}\right|-1} f(j), \quad \cdots n_{77}+1 \leqslant\left|n_{2}\right| \leqslant n_{75}+n_{77},
\end{array}\right.
\end{gathered}
$$

while $g\left(n_{2}\right)=f\left(n_{2}\right)=0$ when $\left|n_{2}\right| \leqslant n_{77}$. In Eq. (2), $\gamma_{D}(i ; j) C_{1}$ and $\gamma_{A B}(i ; j) C_{2}$ derive from the direct flux and $A B$ flux, respectively. Equation (2) itself satisfies the condition that $\phi_{0} B \sum_{i=1}^{j-1} \beta\left(n^{(i+1)} ; n^{(i)}\right) /(2 \pi)$ must be equal to the magnetic flux bounded by the closed loop $n^{(1)} \rightarrow n^{(2)} \rightarrow \cdots n^{(j)}=n^{(1)}$.

\section{RESULTS}

The origin of GOP in a circular CNT torus can be explained using the dispersion relation $E_{l}(k, B)$ for a straight CNT under a uniform magnetic field $B$ applied perpendicular to the tube axis. Ajiki and Ando showed that the direct flux flattens the dispersion lines near the Fermi level. ${ }^{21}$ This is shown schematically by the dashed curves $E_{l}(k, B)$ compared to the linear dispersion $E_{l}(k, 0)$ in Fig. 3. Neglecting lattice distortion, the energy level of the circular CNT torus $\varepsilon_{l, j}$ can be expressed by

$$
\begin{gathered}
\epsilon_{l, j}=E_{l}\left(k_{j}(B), B\right), \\
k_{j}(B)=\left\{j+\left(B S_{A B} / \phi_{0}\right)\right\} / R,
\end{gathered}
$$

where $S_{A B}$ is the area of the inner hole and $j$ is an integer. Equation (6) represents the $A B$ effect, while the difference between $E_{l}\left(k_{j}(B), B\right)$ and $E_{l}\left(k_{j}(B), 0\right)$ originates from the direct flux. The total energy $U(B)$ is then obtained as

$$
U(B)=2 \sum_{l, j} f_{\mathrm{FD}}\left(\epsilon_{l, j}\right) \epsilon_{l, j},
$$

where $f_{\mathrm{FD}}$ is the Fermi-Dirac distribution function and the factor of 2 represents spin degeneracy. The Zeeman effect is neglected here but will be discussed later. Considering the case of absolute zero temperature, $\Sigma_{l, j} f_{\mathrm{FD}}$ can be replaced by the summation $\Sigma_{l, j=\text { occ }}$ limited to the occupied states.

The magnetic moment per torus $M$ is calculated by $M=-d U / d B$. The effect of direct flux can be isolated by extracting the magnetic moment induced solely by $A B$ flux, as follows:

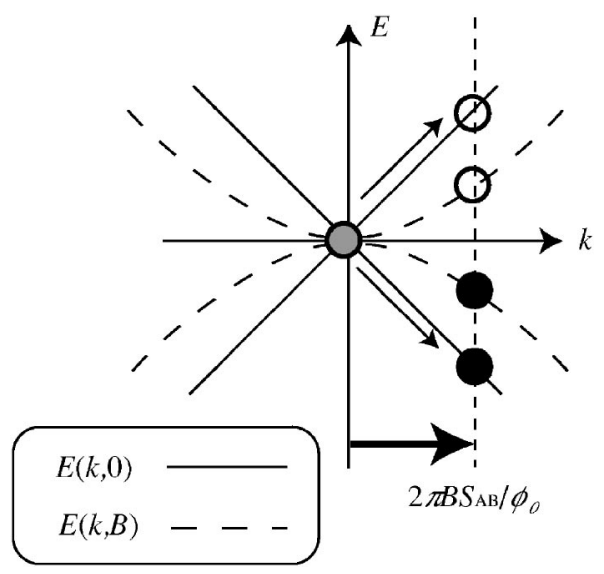

FIG. 3. Dispersion relation with (dashed) and without (solid) direct flux. In the absence of a magnetic field $(B=0)$, the HOL of the torus is located at the crossing of the solid lines (gray circle). The HOL is degenerate and partially occupied. Under finite $B$, the degeneracy is lifted and only the lower level (closed circle) is occupied, while the higher level becomes vacant (open circle). The change of level induced by the $A B$ effect is indicated by an arrow. 
TABLE I. The band structure of periodic CNT junctions, $E_{N}(\alpha, 0)$ and $E_{N+1}(\alpha, 0)$.

\begin{tabular}{|c|c|c|c|c|}
\hline \multicolumn{5}{|c|}{$n_{75} / 3=$ integer? } \\
\hline \multicolumn{2}{|c|}{ Yes } & \multicolumn{3}{|c|}{ No } \\
\hline \multicolumn{2}{|c|}{$n_{S} / 3=$ integer? } & \multicolumn{3}{|c|}{$n_{S} / 3=$ integer $?$} \\
\hline \multirow[t]{3}{*}{ Yes } & \multirow[t]{3}{*}{ No } & \multirow[t]{3}{*}{ Yes } & \multicolumn{2}{|c|}{ No } \\
\hline & & & \multicolumn{2}{|c|}{$\left(n_{S}-n_{75}\right) / 3=$ integer? } \\
\hline & & & Yes & No \\
\hline Type 1 & Type 2 & Type 3 & Type 4-1 & Type 4-2 \\
\hline \multirow[t]{3}{*}{ Semiconductor } & Metal & Semimetal & Semimetal & Semiconductor \\
\hline & & Semiconductor & Semiconductor & \\
\hline & & Metal & Metal & \\
\hline
\end{tabular}

$$
M_{A B}=-S_{A B} /\left.\left(R \phi_{0}\right) \sum_{l, j=\mathrm{occ}}\left(d E_{l}(k, 0) / d k\right)\right|_{k=k_{j}(0)} .
$$

Then, since $E_{l}(k, 0)$ is an even function of $k$, the energy levels at $k=k_{j}(0)$ and $k=k_{-j}(0)=-k_{j}(0)$ are occupied at the same time. Their contribution to $M_{A B}$ usually cancels out because $d E_{l}(k, 0) / d k$ is an odd function of $k$. However, when the highest occupied level (HOL) corresponds to the $K$ and $K^{\prime}$ corner points, this cancellation does not occur and GOP is induced. This is illustrated in Fig. 3, where the energy levels and the change caused by the $A B$ effect are indicated by circles and arrows, respectively. Without direct flux, the levels move along the linear dispersion line and only the level with negative $d E / d k$ is occupied (closed circle), while the level with positive $d E / d k$ becomes vacant (open circle). Only the former then contributes to the magnetic moment, giving rise to GOP. The exclusive occupation of the state with negative $d E / d k$ corresponds to the generation of persistent current. The essential finding here is that the corner point is only partially occupied (gray circle). If the corner point were to be fully occupied, the level indicated by the open circle would also be occupied and the energy decrease of the closed-circle level would be canceled. Under direct flux, however, the closed-circle level is located on the dashed curve and is shifted upward compared to the case without direct flux, that is, direct flux suppresses GOP.

The magnetic moment of the polygonal torus can be calculated by an almost identical approach as for the circular torus. In this case, the phase $\alpha$ is used instead of the crystal wave number $k$. Rotation by $\pi / 3$ with respect to the sixfold symmetry axis is equivalent to multiplying the wave function by the phase factor $\exp (i \alpha)$, as shown in Fig. 1. Although $k \pi R / 3$ corresponds to $\alpha$, it should be noted that $R$ cannot be defined uniquely for the polygonal torus. The energy levels $E_{l}(\alpha)$ are the eigenvalues of the matrix $H_{1}(B)$ $+\exp (i \alpha) H_{2}(B)+\exp (-i \alpha) H_{2}^{\dagger}(B)$, where $H_{1}$ and $H_{2}$ refer to the bonds in the unit cell and those connecting the neighboring unit cells, respectively. These values can be obtained from the Hamiltonian matrix $H$ as $H_{1}\left(n_{2}, n_{3} ; m_{2}, m_{3}\right)$ $\equiv H\left(n_{1}, n_{2}, n_{3} ; n_{1}, m_{2}, m_{3}\right), \quad$ and $\quad H_{2}\left(n_{2}, n_{3} ; m_{2}, m_{3}\right)$ $\equiv H\left(n_{1}, n_{2}, n_{3} ; n_{1}+1, m_{2}, m_{3}\right)$, with the atom labeling $\left(n_{1}, n_{2}, n_{3}\right)$ as defined above. As the wave function is invariant under $2 \pi$ rotation, $\alpha$ takes discrete values $\alpha_{j}=(\pi / 3)(j$ $\left.+\left(B S_{A B} / \phi_{0}\right)\right) \quad(j=$ integer $)$ including the $A B$ flux, $B S_{A B}$ $=B 3 \sqrt{3}\left|\vec{S}_{2}\right|^{2} / 2$. This results in discrete levels of $E_{l}\left(\alpha_{j}, B\right)$ for the CNT torus. Alternatively, $E_{l}(\alpha, B)$ with continuous $\alpha$ represents the continuous energy spectra of the periodic CNT junction formed by connecting unit cells of the CNT torus.

The band structure $E_{l}(\alpha, 0)$ in the present analysis is classified as listed in Table I using three parameters, $n_{S} / 3, n_{75} / 3$, and $\left(n_{S}-n_{75}\right) / 3$, after Ref. 14. As shown in Fig. 2, the CNT torus contains two kinds of CNTs, the original armchair CNT with chiral vector $\vec{L}$, and another characterized by a different chiral vector $\vec{L}^{\prime}$. When $n_{75} / 3$ is an integer, the latter becomes also metallic. It has been proved analytically that types 1 and 2 are semiconducting and metallic, respectively. ${ }^{15}$ Although only numerical results have been obtained for other types, it can be concluded that the CNTs tend to become semiconducting as the axial length of the semiconducting CNT segment $\left|\vec{L}^{\prime} \times \vec{R}_{77}\right| /\left|\vec{L}^{\prime}\right|$ increases. $^{22}$

The dispersion curves for a type- 2 periodic CNT junction (metallic) and a type-3 periodic CNT junction (semimetallic) are shown in Figs. 4 and 5. The index $l$ of $E_{l}(\alpha, B)$ is defined as $E_{1}(\alpha, B) \leqslant E_{2}(\alpha, B) \leqslant \cdots, \leqslant E_{2 N}(\alpha, B)$, where $2 N$ is the number of atoms in the unit cell, that is, $N \equiv 2\left(n_{S} n_{L}\right.$ $\left.-n_{57} n_{77}\right)-n_{57}^{2}$. Thus, the HOMO band is denoted by $E_{N}(\alpha, B)$ and $E_{N+1}(\alpha, B)$ corresponds to the LUMO band.

The magnetic moment exclusively under $A B$ flux $M_{A B}$ is analogous to Eq. (8) as follows:

$$
M_{A B}=-\left.C_{2} \sum_{l, j=\mathrm{occ}}\left(d E_{l}(\alpha, 0) / d \alpha\right)\right|_{\alpha=\alpha_{j}},
$$

where $C_{2}$ is as defined in Eq. (2). The HOMO and LUMO bands of type 2 are similar to those of metallic CNTs, but the band crossing point is shifted slightly from $\alpha= \pm 2 \pi / 3$ as a result of phase shift at the disclinations. ${ }^{15}$ Therefore, the HOL is fully occupied and GOP does not occur. Under finite $B$, however, $\alpha=2 \pi / 3+C_{2} B$ arises at the crossing point such that $M_{A B}$ exhibits a discrete change from negative to positive, as shown in Fig. 6. In contrast, the HOL can be partially occupied in type-3 and 4-1 CNT tori under the condition that 


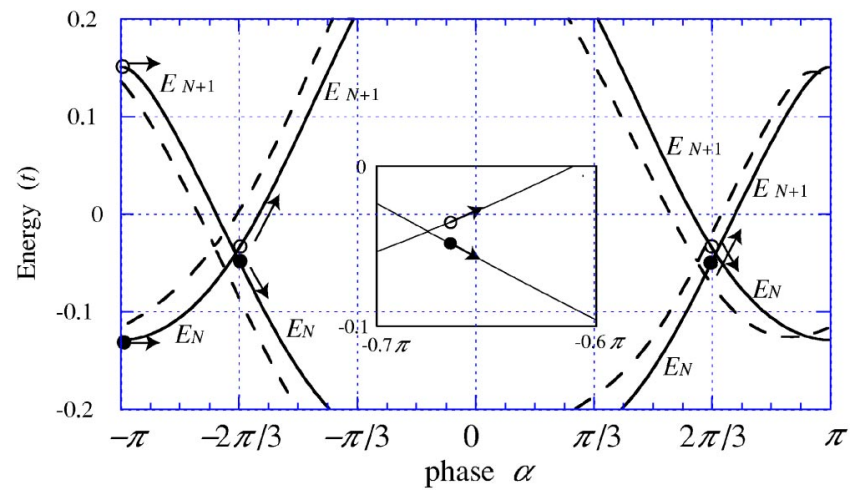

FIG. 4. Band structure of type-2 periodic CNT junction with parameters $\left(n_{S}, n_{L}, n_{75}, n_{55}\right)=(5,7,3,2)$. Circles, lines, and arrows have the same meanings as in Fig. 3. Dashed lines denote the condition $B=0.01 \phi_{0} / a^{2}$. (Inset) Detail of the band structure near the $\operatorname{HOL}\left(E_{N}(-2 \pi / 3,0)\right)$.

the corresponding periodic CNT junction is semimetallic. Figure 5 shows an example of this scenario, where $E_{N}$ at $\alpha$ $=0$ is vacant and $E_{N+1}$ at $\alpha= \pm \pi / 3$ is partially occupied. The $A B$ flux lifts the degeneracy of the HOLs, leaving only the lowered level at $\alpha=\pi / 3$ occupied, thus allowing GOP.

How, then, does direct flux affect GOP? Surprisingly, direct flux enhances GOP. The dashed lines in Fig. 5 represent the dispersion relation $E_{l}(\alpha, B)$ under a finite magnetic field of $B=0.01 \phi_{0} / a^{2}$. In contrast to Fig. 3, the dispersion lines are shifted along the $\alpha$ axis rather than along the $E$ axis. This horizontal shift, rather than canceling the effect of the $A B$ shift, in fact enhances it. Here it should be noted that there is competition between GOP and spin paramagnetism. When Zeeman splitting of $g \mu_{B} B$ ( $\mu_{B}$ is the Bohr magneton and $g$ $\simeq 2$ ) is larger than the GOP splitting of $|M B|=|B d E / d B|$, both HOLs with positive $d E / d \alpha$ and negative $d E / d \alpha$ become occupied by the same spin. This gives rise to a spin magnetic moment of $2 g \mu_{B}$ per torus instead of GOP. As $\mu_{B} \simeq t a^{2} / \phi_{0}(t \simeq 3 \mathrm{eV}, a \simeq 0.25 \mathrm{~nm})$, GOP is relevant only when $M$ is larger than $4 t /\left(\phi_{0} / a^{2}\right)$. To search for the polygonal CNT torus exhibiting GOP in this context, the magnetic moment $M(1.5 \Delta B)=-(U(2 \Delta B)-U(\Delta B)) / \Delta B$ with $\Delta B=0.5$

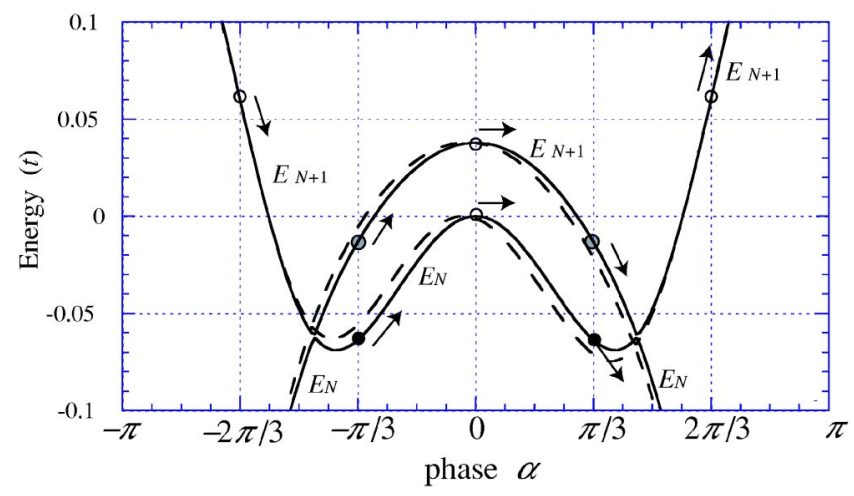

FIG. 5. Band structure of type-3 periodic CNT junction with parameters $\left(n_{S}, n_{L}, n_{75}, n_{55}\right)=(3,7,1,3)$. Circles, lines, and arrows have the same meanings as in Fig. 3. Dashed lines denote the condition $B=0.01 \phi_{0} / a^{2}$.

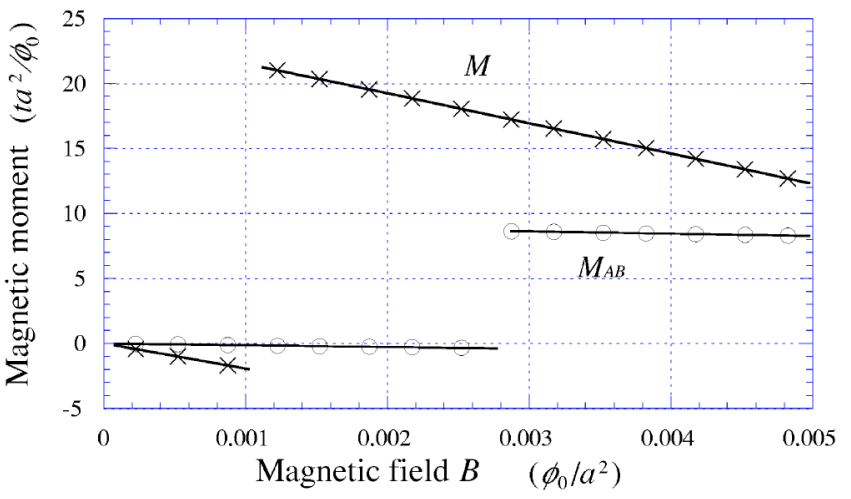

FIG. 6. Magnetic moments of the polygonal tori corresponding to Fig. 4 (i.e., $\left.\left(n_{S}, n_{L}, n_{75}, n_{55}\right)=(5,7,3,2)\right)$ as a function of magnetic field $B . M$ and $M_{A B}$ denote the magnetic moments induced by full flux and $A B$ flux only. The full-flux magnetic moment is calculated from the total energy $U(B)$ by $M((j-0.5) \Delta B)=-(U(j \Delta B)$ $-U((j-1) \Delta B)) / \Delta B \quad$ with $\quad \Delta B=0.5 \times 10^{-4}\left(\phi_{0} / a^{2}\right)$ and $j$ $=2,3, \ldots, 100$. For $M_{A B}$, the total energy is calculated from the Hamiltonian with $\gamma^{D}$ in Eq. (2) is replaced with zero.

$\times 10^{-5} \phi_{0} / a^{2}$ (i.e., $B=1.5 \Delta B \simeq 0.24$ Tesla) was calculated for 207 tori in the parameter ranges of $3 \leqslant n_{S} \leqslant 12,1 \leqslant n_{75}$ $\leqslant 6,3 \leqslant n_{L} \leqslant 12$ and $1 \leqslant n_{77}=\left(n_{L}-n_{75}\right) / 2 \leqslant 6$. Among these tori, only 15 had $M$ larger than $4 t a^{2} / \phi_{0}$, and all belonged to type 3 or type 4-1 and exhibited GOP. Two of the CNT tori displayed $M$ values of larger than $10 \operatorname{ta}^{2} / \phi_{0}$. In contrast, the tori without GOP had $M$ values much lower than $4 t a^{2} / \phi_{0}$. Since $M B$ approximately corresponds to the splitting between the degenerate HOLs induced by $B$, the temperature must be lower than $M B$ for GOP to occur. When $M$ $=10 t /\left(\Phi_{0} / a^{2}\right)$ and $B=1.5 \Delta B, M B \sim 2 \mathrm{~K}$, which is experimentally achievable.

The distribution of $M$ and its enhancement by direct flux are shown in Fig. 7, where $M_{A B} / M$ is plotted as a function of $M$ for the tori exhibiting GOP. For all tori, $M_{A B}<M$, that is, direct flux enhances GOP. The effect of direct flux can be almost entirely included in the $A B$ effect by assuming a cross

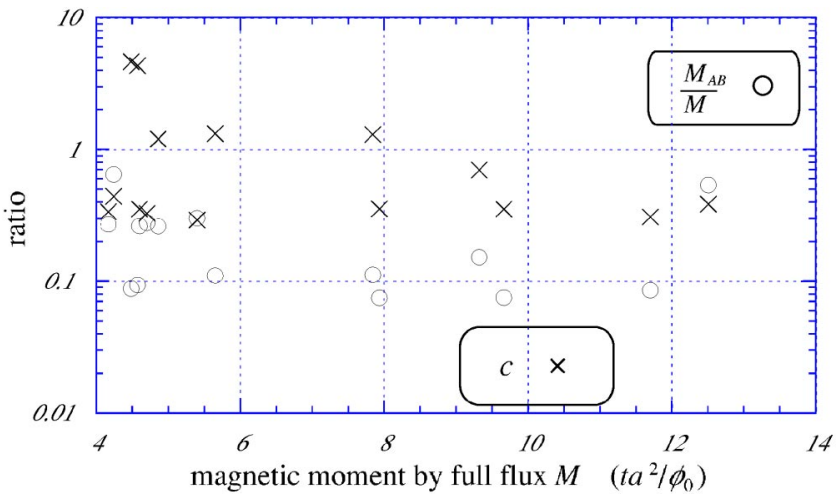

FIG. 7. Ratios $M_{A B} / M$ and $c=\left[\left(M-M_{A B}\right) / M_{A B}\right]\left[\left|\vec{S}_{2}\right|^{2} /\left(|\vec{S}|^{2}\right.\right.$ $\left.\left.-\left|\vec{S}_{2}\right|^{2}\right)\right]$ as functions of $M$ for $15 \mathrm{CNT}$ tori with $M>4 t a^{2} / \phi_{0}$, where $M$ and $M_{A B}$ denote the magnetic moments due to full flux and $A B$ flux only. The applied magnetic field $B$ is 0.75 $\times 10^{-5} \phi_{0} / a^{2}$. The factor $|\vec{S}|^{2} /\left|\vec{S}_{2}\right|^{2}$ denotes the ratio of full flux to $A B$ flux. 
section of $c S_{D}+\left(3 \sqrt{3}\left|\vec{S}_{2}\right|^{2} / 2\right)$ rather than $3 \sqrt{3}\left|\vec{S}_{2}\right|^{2} / 2$, using the cross section of the graphite plane $S_{D}=3 \sqrt{3}\left(|\vec{S}|^{2}\right.$ $\left.-\left|\vec{S}_{2}\right|^{2}\right) / 2$. Relation between $c$ and $M_{A B} / M$ are represented by

$$
c=\left(\frac{M-M_{A B}}{M_{A B}}\right)\left(\frac{\left|\vec{S}_{2}\right|^{2}}{|\vec{S}|^{2}-\left|\vec{S}_{2}\right|^{2}}\right) .
$$

This is equivalent to replacing $C_{1}$ and $C_{2}$ in Eq. (2) with $C_{1}=0 \quad$ and $\quad C_{2}=\sqrt{3} \pi\left|\vec{S}_{2}\right|^{2} /\left(2 \phi_{0}\right)+\pi c S_{D} /\left(3 \phi_{0}\right)$. In other words, the HOMO and LUMO dispersion curve can be approximated by

$$
E_{l}(\alpha, B) \simeq E_{l}\left(\alpha+(c \pi / 3)\left(B S_{D} / \phi_{0}\right), 0\right),
$$

where $c S_{D}$ represents the effective cross section of the graphite plane contributing to the $A B$ effect. Since Fig. 7 shows that values of $c$ are comparable to unity, we can say that $M$ is close to that calculated based on the Aharonov-Bohm alone for a cross section that includes the region of direct flux. The shift of $\alpha$ in Eq. (11) can in fact be seen in Figs. 4 and 5, where the dashed lines represent the dispersion relation $E_{l}\left(\alpha, B=0.01 \phi_{0} / a^{2}\right)$. Equation (11) also indicates that the magnetic moment $M$ undergoes discrete changes at $B$ lower than $M_{A B}$ Fig. 6.

\section{SUMMARY AND DISCUSSION}

In some of the polygonal CNT tori examined in this study, persistent current leads to a large positive magnetic suscep- tibility that varies in inverse proportion to temperature. This phenomenon is referred to as giant orbital paramagnetism (GOP) in this paper. It was shown that the magnetic moment per torus can be larger than the Bohr magneton under achievable conditions of $B \sim 0.24$ Tesla and $T<2 \mathrm{~K}$. For GOP to occur, the corresponding periodic CNT junction must be semimetallic (i.e., type 3 or type 4-1). The magnetic flux was separated into $A B$ flux in the inner hole and direct flux intersecting the graphite plane, and it was found that despite the diamagnetism of graphite, direct flux in fact enhances GOP and its effect can be effectively included in the $A B$ effect by assuming a cross section that encompasses the area bounded by the outer fringe rather than the inner hole area alone, as shown by Eq. (11) and in Fig. 7.

GOP in circular CNT tori is considered to be caused by the metallic band structure of the straight CNT, whereas GOP in polygonal CNT tori originates from the semimetallic band structures of the periodic CNT junction. This difference originates from the differing bond networks, that is, removal of the shaded areas in the projection map and connecting the edges (Fig. 2). In comparison, elastic deformation does not affect the bonding of $n$ and $m$ and as such is irrelevant with respect to GOP in polygonal CNT tori. The discovery of this new kind of GOP highlights the potential of polygonal CNT tori as a nanostructure capable of large persistent current.

\section{ACKNOWLEDGMENT}

This work was supported in part by a Grant-in-Aid for Creative Scientific Research on "Devices on molecular and DNA levels" (No. 13GS0017) from the Japan Society for the Promotion of Science.
${ }^{1}$ N. Hamada, S. I. Sawada, and A. Oshiyama, Phys. Rev. Lett. 68, 1579 (1992); J. W. Mintmire, B. I. Dunlap, and C. T. White, ibid. 68, 631 (1992); R. Saito, M. Fujita, G. Dresselhaus, and M. S. Dresselhaus, Phys. Rev. B 46, 1804 (1992).

${ }^{2}$ D. Loss and P. Goldbart, Phys. Rev. B 43, 13762 (1991).

${ }^{3}$ H. F. Cheung, Y. Gefen, E. K. Riedel, and W. H. Shih, Phys. Rev. B 37, 6050 (1988).

${ }^{4}$ M. F. Lin and D. S. Chuu, Phys. Rev. B 57, 6731 (1998).

${ }^{5}$ S. J. Tans and C. Dekker, Nature (London) 385, 780 (1997); R. Martel, H. R. Shea, and P. Avouris, ibid. 398, 780 (1997); H. R. Shea, R. Martel, and P. Avouris, Phys. Rev. Lett. 84, 4441 (2000); M. Ahlskog, E. Seynaeve, R. J. M. Vullers, C. Van Haesendonck, A. Fonseca, K. Hernadi, and J. B. Nagy, Chem. Phys. Lett. 300, 202 (1999).

${ }^{6}$ H. Ajiki and T. Ando, J. Phys. Soc. Jpn. 62, 2470 (1993); 63, 4267 (1994).

${ }^{7}$ L. P. Levy, G. Dolan, J. Dunsmuir, and H. Bouchiat, Phys. Rev. Lett. 64, 2074 (1990); V. Chandrasekhar, R. A. Webb, M. J. Brady, M. B. Ketchen, W. J. Gallagher, and A. Kleinsasser, ibid. 67, 3578 (1991); D. Mailly, C. Chapelier, and A. Benoit, ibid. 70, 2020 (1993).

${ }^{8}$ S. Latil, S. Roche, and A. Rubio, Phys. Rev. B 67, 165420 (2003).

${ }^{9}$ A. Latgé, C. G. Rocha, L. A. L. Wanderley, M. Pacheco, P. Orel- lana, and Z. Barticevic, Phys. Rev. B 67, 155413 (2003).

${ }^{10}$ V. Meunier, P. Lambin, and A. A. Lucas, Phys. Rev. B 57, 14886 (1998).

${ }^{11}$ C. C. Tsai, F. L. Shyu, C. W. Chiu, C. P. Chang, R. B. Chen, and M. F. Lin, Phys. Rev. B 70, 075411 (2004); K. Sasaki, ibid. 65, 155429 (2002); L. Liu, G. Y. Guo, C. S. Jayanthi, and S. Y. Wu, Phys. Rev. Lett. 88, 217206 (2002); A. A. Oditsov, W. Smit, and H. Yoshioka, Europhys. Lett. 45, 598 (1999).

${ }^{12}$ R. Tamura, Phys. Rev. B 67, 121408(R) (2003); 64, 201404(R) (2001); R. Tamura and M. Tsukada, ibid. 61, 8548 (2000); H. Matsumura and T. Ando, J. Phys. Soc. Jpn. 67, 3542 (1998); L. Chico, V. H. Crespi, L. X. Benedict, S. G. Louie, and M. L. Cohen, Phys. Rev. Lett. 76, 971 (1996).

${ }^{13}$ R. Tamura and M. Tsukada, Phys. Rev. B 52, 6015 (1995); Y. Kasahara, R. Tamura, and M. Tsukada, ibid. 67, 115419 (2003); T. Yaguchi and T. Ando, J. Phys. Soc. Jpn. 70, 1327 (2001).

${ }^{14}$ K. Akagi, R. Tamura, M. Tsukada, S. Itoh, and S. Ihara, Phys. Rev. B 53, 2114 (1996).

${ }^{15}$ R. Tamura and M. Tsukada, J. Phys. Soc. Jpn. 68, 910 (1999).

${ }^{16}$ S. Itoh and S. Ihara, Phys. Rev. B 48, 8323 (1993); B. I. Dunlap, ibid. 46, 1933 (1992); D. H. Oh, J. M. Park, and K. S. Kim, ibid. 62, 1600 (2000).

${ }^{17}$ R. C. Haddon, Nature (London) 388, 31 (1997).

${ }^{18}$ L. Yang, J. Jiang, and J. Dong, Phys. Status Solidi B 238, 115 
(2003); S. Zhang, S. Zhao, M. Xia, E. Zhang, and T. Xu, Phys. Rev. B 68, 245419 (2003).

${ }^{19}$ O. Hod, E. Rabani, and R. Baer, Phys. Rev. B 67, 195408 (2003); M. Huhtala, A. Kuronen, and K. Kaski, Comput. Phys. Commun. 147, 91 (2002).

${ }^{20}$ R. Tamura and M. Tsukada, Phys. Rev. B 49, 7697 (1994); R.
Tamura, K. Akagi, M. Tsukada, S. Itoh, and S. Ihara, ibid. 56, 1404 (1997).

${ }^{21}$ H. Ajiki and T. Ando, J. Phys. Soc. Jpn. 65, 505 (1996).

${ }^{22}$ Although the periodic CNT junctions of types 3 and 4-1 were reported to be semimetallic in Ref. 14, it was subsequently found later both types may also be semiconducting and metallic. 